\title{
Comparative Effect of Ultrasound Therapy and Laser Therapy for Relief of Pain, Swelling and Trismus Following Third Molar Surgeries
}

\author{
Sukhdeep Kaur ${ }^{1}$, Apoorv Narain ${ }^{2}$, Vinod Kapoor ${ }^{3}$, Jagmohan Singh ${ }^{4}$ \\ ${ }^{1}$ MPT 2nd Year Student, ${ }^{2}$ Associate Professor, ${ }^{3}$ Professor and Principal, Gian Sagar \\ College of Physiotherapy, ${ }^{4}$ Principal and HOD (Oral and Maxillofacial Surgery deptt.), \\ Gian Sagar Dental College and Hospital, Rajpura, Dist. Patiala, Punjab (INDIA)
}

\begin{abstract}
Abstrac: Inability to open the mouth even within its normal limits is called a trismus. This is so due to a reflex spasm of the masticatory muscles leading to a completely or incompletely limited mouth opening in a patient. It is diagnosed from clinical examination of the maximal interincisal distance (MID) of less than $40-45 \mathrm{~mm}$ caused by contracture not by obstructive joint impingement. Objectives of the study was to evaluate the comparative effectiveness of ultrasound therapy \& laser therapy in addition to mouth opening exercises in patients with pain, swelling and trismus following third molar surgeries. 30 subjects aged 18-35 yeras were made part of the study based on inclusion and exclusion criteria and then divided into two groups named group $A$ and group B. Group A received ultrasound therapy for frequency of $1 \mathrm{MHz}$, pulsed $20 \%$ and dose $1.0 \mathrm{~W} / \mathrm{sq} . \mathrm{cm}$ for 7 minutes for first three days after surgery. Group B received laser therapy as output power $15 \mathrm{~mW}$, pulsed $80 \%$ and dose $4.3 \mathrm{~J} / \mathrm{sq}$.cm for 6minutes for also first three days after surgery. Material used in study were Sliding Caliper, Graduated tape, Wooden splints. Pain-It will be evaluated by using VAS scale. Swelling-It will be measured by measuring in between horizontal and vertical directions. Horizontal- It will be measured as horizontal distance from tip of tragus to ipsilateral commissure of mouth.Vertical- It will be measured from lateral external canthus of eye to ipsilateral gonion.Trismus- It will be measured of interincisal distance. Additionally mouth opening exercises both active and passive were given to all the patients in both the groups. Pre and post treatment readings were taken of pain, swelling and trismus. Results showed that there was a significant difference between pain, swelling and trismus within the groups. But there was not a significant difference between both the groups. The study concluded that though statistically both groups showed the significant improvement in all parameters. But when we compare both groups with each other then there is no statistical difference between them. So null hypothesis was accepted.
\end{abstract}

\section{Introduction}

The third molar extraction is one of the most common surgical procedures in oral surgery. After a surgical trauma, an inflammatory process usually appears accompanied 
with pain, trismus and facial edema. These symptoms can affect quality of life of the patients during the first days of the postoperative process.(Sato FR et al 2009 and Colorado et al 2006). A wide variety of analgesics, non-steroidal anti-inflammatory drugs (NSAIDs), local or systemic corticosteroids, long-term anesthetics, etc., designed to control these complications.(Vegas-Bustamante et al 2008). However, these drugs are unsafe and can sometimes induce side-effects. Hence, there is a growing interest in developing alternative or complementary methods free of adverse effects.(Merry AF et al. 2010). The most severe pain usually occurs during the first 3-5 hrs after the disappearance of the effect of the local anesthesia.(Fisher SE et al 1988). Swelling in contrast, usually reaches its peak between the first 24-48 hrs and gradually decreases in the following days to completely disappear between 5 and 7 days later. As the swelling subside, trismus decreases.(Berge TI et al 1994). Low power laser (LPL) induces primary (photochemical, photoelectrical, and photoenergetic) and secondary (stimulation of cell metabolism and microcirculation) biostimulation potential and reduces pain and edema after surgery.(Miserendino et al.1995). As there is no ideal means of prevention the postoperative trismus yet, it appears that the application of LPL in certain postoperative period could contribute to the more successful and faster recovery of a patient, especially considering that there are no adverse effects of irradiation.The biologic effect of different types of ultrasound was tested, and it was concluded that the use of the non-invasive low intensity pulsed ultrasound (LIPUS) has an optimal biologic effect in promoting tissue healing.(Tanzer et al 1996).

\section{Need of study}

The treatment of trismus over the past few years have been varied, which includes medical \& surgical management but with unpredictable results. So for the betterment of the patients symptoms the role of physiotherapy treatment to enhance the speedy recovery of the patients needs to be further evaluated. Since the data lacks concrete evidence for the use of ultrasound in trismus, study is needed to monitor the effect of the same.

Aim of the study

To compare the effectiveness of ultrasound therapy and laser therapy with pain, swelling and trismus following third molar surgeries.

Objectives of the study

To compare the effect of ultrasound therapy and laser therapy for relief of pain, swelling and trismus following third molar surgeries.

To analyse the effect of ultrasound therapy for relief of pain, swelling and trismus following third molar surgeries.

To analyse the effect of laser therapy for relief of pain, swelling and trismus following third molar surgeries.

Hypothesis

Null hypothesis 
There will be no significant difference in the effect of ultrasound therapy and laser therapy with mouth opening exercises in patients with pain, swelling and trismus in third molar surgeries.

Alternate hypothesis

There will be a significant difference in the effect of ultrasound therapy and laser therapy with mouth opening exercises in patients with pain, swelling and trismus in third molar surgeries.

Study design- Prospective, randomized, comparative and an experimental study design.

Sample- 30 subjects

Sampling technique- Random sampling technique

Inclusion criteria-

Patients were included in the present prospective randomized study irrespective of sex, caste, religion and socio-economic status.

Patients of age group between 16-35yrs.

Patients with post-operative mouth opening is less than equal to $30 \mathrm{~mm}$ on day one.

Exclusion criteria -

Post operative complications like acute sepsis, hemorrhage, etc.

Tumours.

Implants.

Anaesthetic area.

Non-cooperative patients.

Procedure-

A total of 30 patients will be included in the study based on the inclusion and exclusion criteria. These patients will be then randomly divided into two groups that is group I (15 patients) and group II (15 patients). Both groups I and II are under experimental group. Following surgical removal of third molars in the department of oral and maxillofacial surgery by an oral surgeon, group I will receive ultrasound therapy and group II will receive laser therapy as per the protocol. The ultrasound will be given as frequency of $1 \mathrm{MHz}$, pulsed $20 \%$ and dose $1.0 \mathrm{~W} / \mathrm{sq} . \mathrm{cm}$ for 7 minutes for first three days after surgery. The laser will be given as output power $15 \mathrm{~mW}$, pulsed $80 \%$ and dose $4.3 \mathrm{~J} / \mathrm{sq} . \mathrm{cm}$ for 6 minutes for also first three days after surgery. Both applied extraorally near the insertion of the masseter muscle. All the patients in both the groups will be given same pre and post of medication. Additionally mouth opening exercises both active and passive will be given to all the patients in both the groups.

Dependent Variables-

Visual analogue scale.(VAS).

Edema coefficient (Ec)

Trismus coefficient (Tc).

Independent variables

Mouth opening exercises. 
Ultrasound therapy

Laser therapy.

Operational tools

Ultrasound therapy

Laser therapy.

Statistics

Paired t-test will be done between pre and post observations of VAS scale in both groupl and groupll.

Paired t-test will be done between pre and post Edema coefficient(Ec) and Trismus coefficient $(T c)$ in both groupI and groupII

Unpaired t- test to difference between both the group I and group II.

Results

Table 1

Comparison of VAS within both the group

\begin{tabular}{|c|c|c|c|c|c|c|c|c|c|}
\hline \multicolumn{4}{|c|}{ Paired Samples Statistics } & \multicolumn{3}{|c|}{ Paired Differences } & & & \\
\hline \multicolumn{2}{|c|}{$\operatorname{VAS}(N=15)$} & \multirow{2}{*}{$\begin{array}{c}\text { Mean+Std } \\
\text { Deviation } \\
6.74+1.28\end{array}$} & $\begin{array}{c}\text { Std. Error } \\
\text { Mean }\end{array}$ & Mean & \multirow{3}{*}{$\begin{array}{c}\text { Std. } \\
\text { Deviation } \\
1,183\end{array}$} & \multirow{3}{*}{$\begin{array}{c}\text { Std. } \\
\text { Error } \\
\text { Mean } \\
0.306\end{array}$} & \multirow{3}{*}{$\begin{array}{l}t \text { Test } \\
18.33\end{array}$} & \multirow{3}{*}{$\begin{array}{l}d f \\
14\end{array}$} & \multirow{3}{*}{$\begin{array}{c}P \\
\text { Valu } \\
e \\
0.000 \\
\text { SIG }\end{array}$} \\
\hline \multirow{2}{*}{ GROUPA } & $P R E$ & & 0.330 & \multirow{2}{*}{5.60} & & & & & \\
\hline & $P O S T$ & $1.14+0.36$ & 0.091 & & & & & & \\
\hline \multirow[b]{2}{*}{ GROUP B } & $P R E$ & $6.54+1.13$ & 0.291 & \multirow[b]{2}{*}{5.47} & \multirow[b]{2}{*}{1.060} & \multirow[b]{2}{*}{0.274} & \multirow[b]{2}{*}{19.97} & \multirow[b]{2}{*}{14} & \multirow{2}{*}{$\begin{array}{c}0.000 \\
S I G\end{array}$} \\
\hline & POST & $1.07+0.26$ & 0.067 & & & & & & \\
\hline
\end{tabular}

Figure 1.1

Comparison of VAS within both the groups

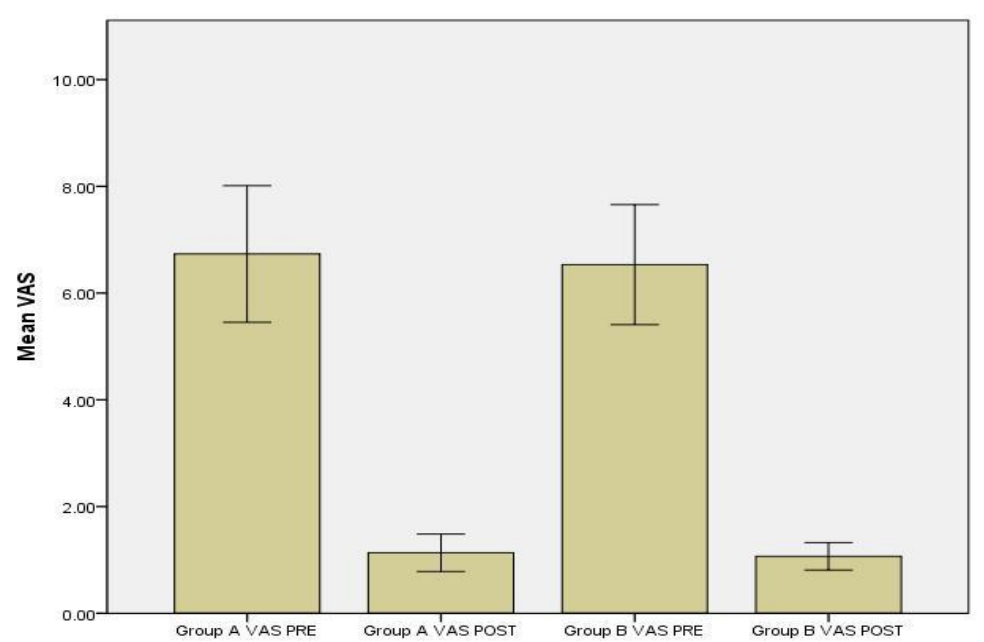


Table 2

Comparison of pre and post swelling in horizontal position within both the groups

\begin{tabular}{|c|c|c|c|c|c|c|c|c|c|}
\hline \multicolumn{4}{|c|}{ Paired Samples Statistics } & \multicolumn{3}{|c|}{ Paired Differences } & & & \\
\hline \multicolumn{2}{|c|}{$\begin{array}{l}\text { SWELLING } \\
\text { HZ(MM) } \\
\mathrm{N}=15\end{array}$} & $\begin{array}{l}\text { Mean+Std } \\
\text { Deviation }\end{array}$ & $\begin{array}{l}\text { Std. } \\
\text { Error } \\
\text { Mean }\end{array}$ & Mean & $\begin{array}{l}\text { Std. } \\
\text { Deviatio } \\
\mathrm{n}\end{array}$ & $\begin{array}{l}\text { Std, } \\
\text { Error } \\
\text { Mean }\end{array}$ & t Test & $\mathrm{df}$ & P Value \\
\hline \multirow{2}{*}{$\begin{array}{l}\text { GRO } \\
\text { UP } \\
\text { A }\end{array}$} & PRE & $112.4+8.254$ & 2.131 & \multirow[b]{2}{*}{4.40} & \multirow[b]{2}{*}{4.421} & \multirow[b]{2}{*}{1.141} & \multirow[b]{2}{*}{3.86} & \multirow[b]{2}{*}{14} & \multirow{2}{*}{$\begin{array}{l}0.002 \\
\text { SIG }\end{array}$} \\
\hline & POST & $108+5.452$ & 1.407 & & & & & & \\
\hline \multirow{2}{*}{$\begin{array}{l}\text { GRO } \\
\text { UP } \\
\text { B }\end{array}$} & PRE & $\begin{array}{l}108.14+4.24 \\
1\end{array}$ & 1.095 & \multirow{2}{*}{\multicolumn{6}{|c|}{ There is no difference between PRE and POST values }} \\
\hline & POST & $\begin{array}{l}108.14+4.24 \\
1\end{array}$ & 1.095 & & & & & & \\
\hline
\end{tabular}

Table 3

Comparison of pre and post swelling in vertical position within both the groups

\begin{tabular}{|c|c|c|c|c|c|c|c|c|c|}
\hline \multicolumn{4}{|c|}{ Paired Samples Statistics } & \multicolumn{3}{|c|}{ Paired Differences } & & & \\
\hline \multicolumn{2}{|c|}{$\begin{array}{l}\text { SWELLING VC(MM) } \\
\qquad \mathrm{N}=15\end{array}$} & $\begin{array}{c}\text { Mean+Std. } \\
\text { Deviation }\end{array}$ & $\begin{array}{l}\text { Std. } \\
\text { Error } \\
\text { Mean }\end{array}$ & Mean & $\begin{array}{c}\text { Std. } \\
\text { Deviatio } \\
n\end{array}$ & $\begin{array}{l}\text { Std. } \\
\text { Error } \\
\text { Mean }\end{array}$ & t Test & $\mathrm{df}$ & $\begin{array}{c}\mathrm{P} \\
\text { Valu } \\
\mathrm{e}\end{array}$ \\
\hline \multirow{2}{*}{$\begin{array}{c}\text { GROUP } \\
\text { A }\end{array}$} & PRE & $111.6+10.12$ & 2.613 & \multirow[b]{2}{*}{4.27} & \multirow[b]{2}{*}{3.283} & \multirow[b]{2}{*}{0.848} & \multirow[b]{2}{*}{5.03} & \multirow[b]{2}{*}{14} & 0.00 \\
\hline & POST & $\begin{array}{c}107.34+8.30 \\
4\end{array}$ & 2.144 & & & & & & $\begin{array}{c}0 \\
\text { SIG }\end{array}$ \\
\hline \multirow{2}{*}{$\begin{array}{c}\text { GROUP } \\
\text { B }\end{array}$} & PRE & $107.87+7.03$ & 1.815 & \multirow{2}{*}{\multicolumn{6}{|c|}{ There is no difference between PRE and POST values }} \\
\hline & POST & $107.87+7.03$ & 1.815 & & & & & & \\
\hline
\end{tabular}

Figure 2.1

Comparison of pre and post swelling in horizontal position within the groups

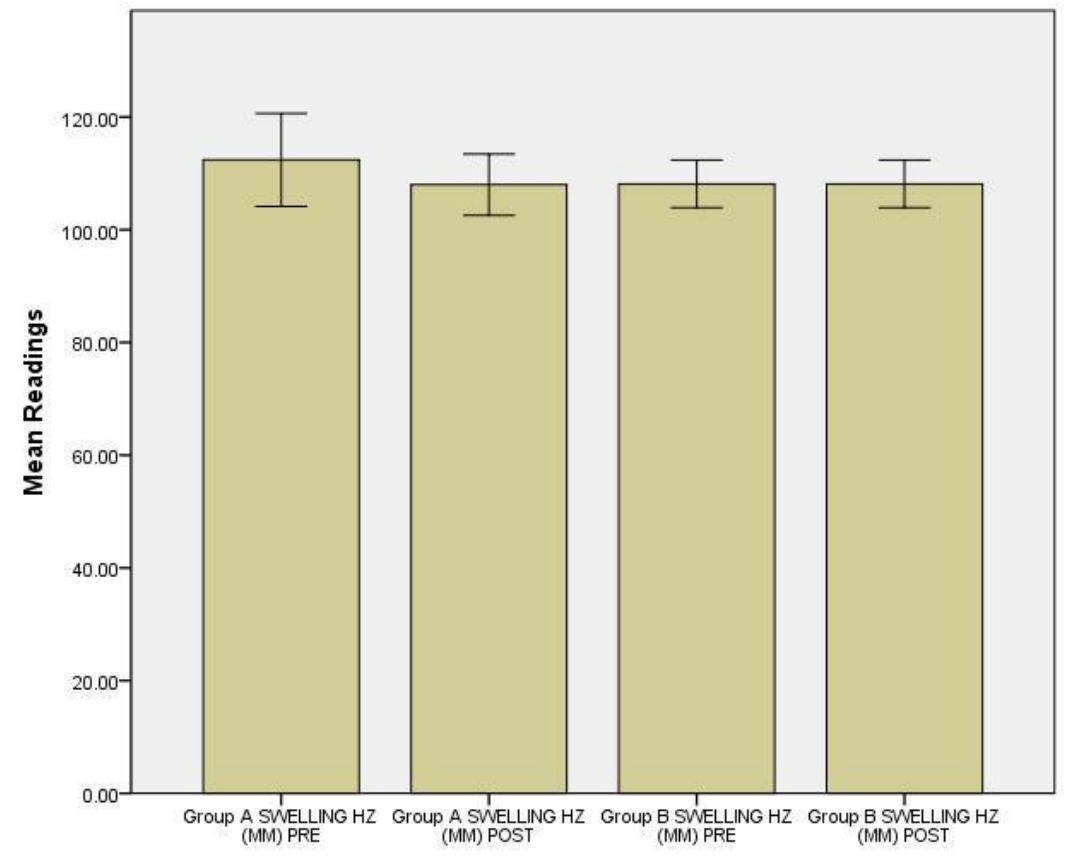


Figure 3.1

Comparison of pre and post swelling in vertical position within the groups

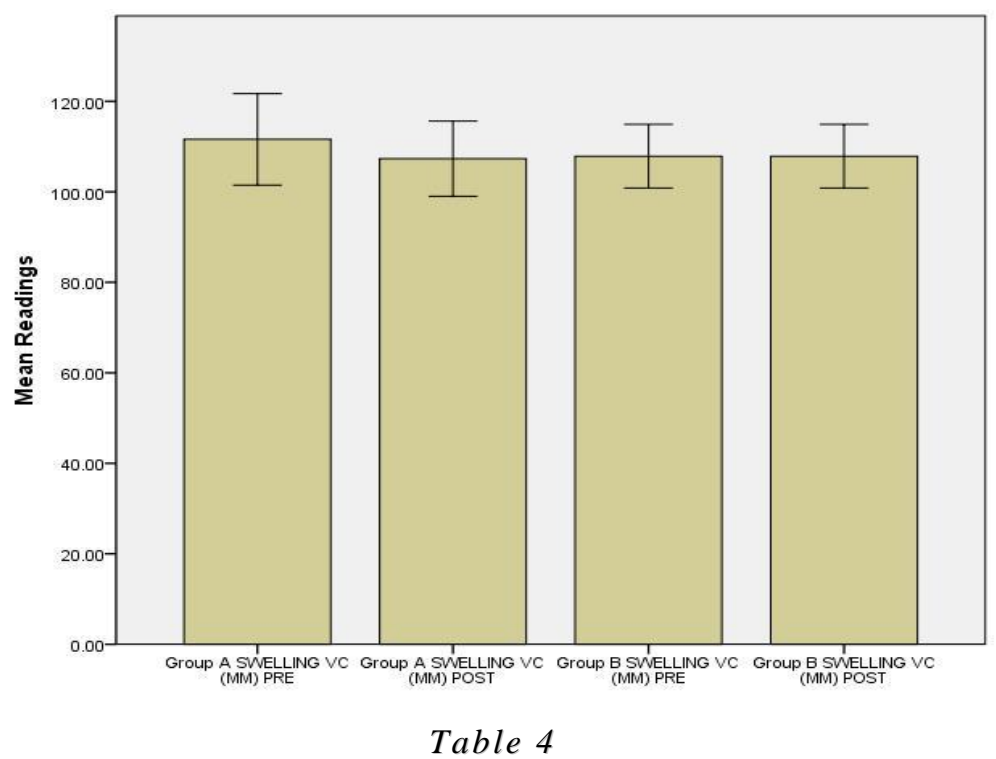

Comparison of trismus within the groups

\begin{tabular}{|c|c|c|c|c|c|c|c|c|c|}
\hline \multicolumn{4}{|c|}{ Paired Samples Statistics } & \multicolumn{3}{|c|}{ Paired Differences } & & & \\
\hline \multicolumn{2}{|c|}{$\begin{array}{c}\text { TRISMUS(MM) } \\
N=15\end{array}$} & $\begin{array}{c}\text { Mean+Std. } \\
\text { Deviation }\end{array}$ & $\begin{array}{l}\text { Std. } \\
\text { Error } \\
\text { Mean }\end{array}$ & Mean & $\begin{array}{c}\text { Std. } \\
\text { Deviat } \\
\text { ion }\end{array}$ & $\begin{array}{l}\text { Std. } \\
\text { Error } \\
\text { Mean }\end{array}$ & $t$ Test & $d f$ & $\begin{array}{c}P \\
\text { Value }\end{array}$ \\
\hline \multirow{2}{*}{$\begin{array}{c}\text { GROU } \\
P A\end{array}$} & $P R E$ & $34.87+7.06$ & 1.823 & \multirow{2}{*}{-6.13} & \multirow{2}{*}{4.969} & \multirow{2}{*}{1.283} & \multirow{2}{*}{-4.78} & \multirow{2}{*}{14} & 0.000 \\
\hline & $P O S T$ & $41+3.163$ & 0.817 & & & & & & $S I G$ \\
\hline \multirow{2}{*}{$\begin{array}{l}G R O U \\
P B\end{array}$} & $P R E$ & $\begin{array}{c}41.74+2.86 \\
6\end{array}$ & 0.740 & \multirow{2}{*}{\multicolumn{6}{|c|}{$\begin{array}{c}\text { There is no difference between } \\
\text { values }\end{array}$}} \\
\hline & $P O S T$ & $\begin{array}{c}41.74+2.86 \\
6\end{array}$ & 0.740 & & & & & & \\
\hline
\end{tabular}

Figure 4.1

Comparison of trismus within the groups

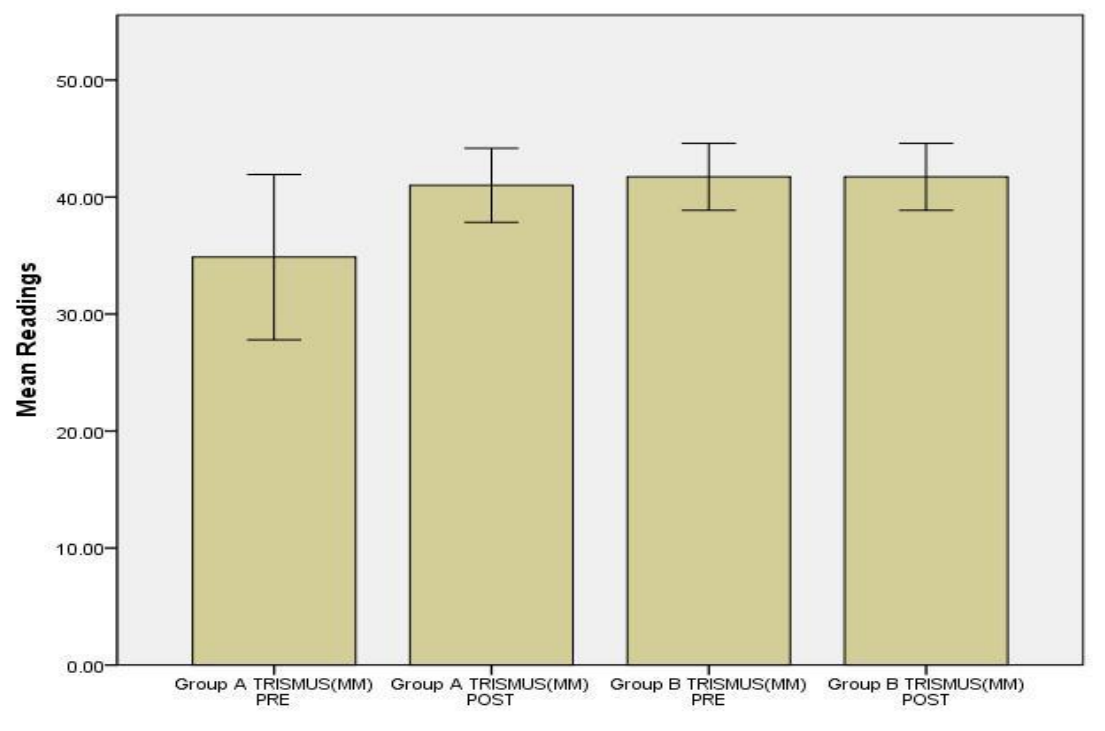


Table 5

Comparison of VAS in between the groups

\begin{tabular}{|c|c|c|c|c|c|c|c|c|}
\hline$N=15$ & GROUP & Mean+Std & $\begin{array}{c}\text { Std. Error } \\
\text { Mean }\end{array}$ & $t$ test & $d f$ & $P$ Value & $\begin{array}{c}\text { Mean } \\
\text { Difference }\end{array}$ & $\begin{array}{c}\text { Std. } \\
\text { Error } \\
\text { Differen } \\
\text { ce }\end{array}$ \\
\hline \multirow{2}{*}{ VAS PRE } & GROUP A & $6.74+1.28$ & 0.330 & \multirow{2}{*}{0.454} & \multirow{2}{*}{28} & \multirow{2}{*}{$0.653 N S$} & \multirow{2}{*}{0.200} & \multirow{2}{*}{0.440} \\
\hline & GROUP B & $6.54+1.126$ & 0.291 & & & & & \\
\hline \multirow{2}{*}{$\begin{array}{c}\text { VAS } \\
\text { POST }\end{array}$} & GROUPA & $1.14+0.352$ & 0.091 & \multirow{2}{*}{0.592} & \multirow{2}{*}{28} & \multirow{2}{*}{$0.559 \mathrm{NS}$} & \multirow{2}{*}{0.067} & \multirow{2}{*}{0.113} \\
\hline & GROUP $B$ & $1.07+0.259$ & 0.067 & & & & & \\
\hline
\end{tabular}

Figure 5.1

Comparison of VAS in between both the groups

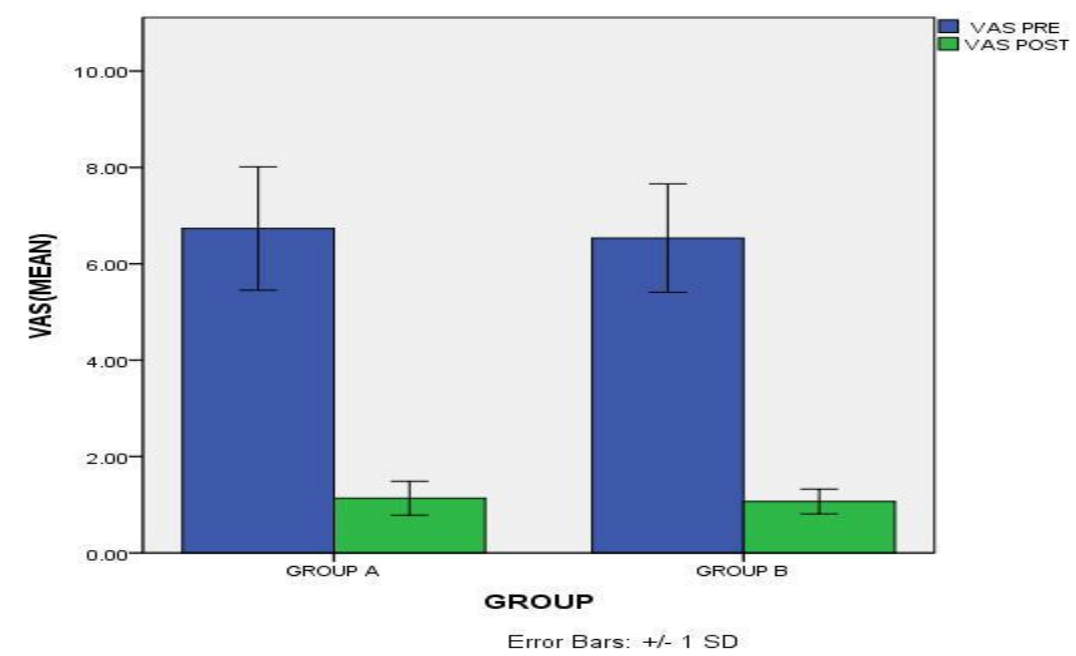

Table 6

Comparison of swelling in horizontal in between both the groups

\begin{tabular}{|c|c|c|c|c|c|c|c|c|}
\hline$N=15$ & GROUP & $\begin{array}{l}\text { Mean+St } \\
d\end{array}$ & $\begin{array}{l}\text { Std. } \\
\text { Error } \\
\text { Mean }\end{array}$ & t test & $d f$ & $P$ Value & $\begin{array}{l}\text { Mean } \\
\text { Differen } \\
\text { ce }\end{array}$ & $\begin{array}{l}\text { Std. } \\
\text { Error } \\
\text { Differen } \\
\text { ce }\end{array}$ \\
\hline \multirow{2}{*}{$\begin{array}{l}\text { SWELLING } \\
H Z(M M) P R E\end{array}$} & $\begin{array}{l}\text { GROUP } \\
A\end{array}$ & $\begin{array}{l}112.4+8 \\
254\end{array}$ & 2.131 & \multirow{2}{*}{1.781} & \multirow{2}{*}{28} & \multirow{2}{*}{$0.086 \mathrm{NS}$} & \multirow{2}{*}{4.267} & \multirow{2}{*}{2.396} \\
\hline & $\begin{array}{l}\text { GROUP } \\
B\end{array}$ & $\begin{array}{l}108.14+4 \\
.241\end{array}$ & 1.095 & & & & & \\
\hline \multirow{2}{*}{$\begin{array}{l}\text { SWELLING } \\
\text { HZ(MM) } \\
\text { POST }\end{array}$} & $\begin{array}{l}\text { GROUP } \\
A\end{array}$ & $\begin{array}{l}108+5.45 \\
2\end{array}$ & 1.407 & \multirow{2}{*}{-0.075} & \multirow{2}{*}{28} & \multirow{2}{*}{$0.941 \mathrm{NS}$} & \multirow{2}{*}{-0.133} & \multirow{2}{*}{1.783} \\
\hline & $\begin{array}{l}\text { GROUP } \\
B\end{array}$ & $\begin{array}{l}108.14+4 \\
.241\end{array}$ & 1.095 & & & & & \\
\hline
\end{tabular}


Figure 6.1

Comparison of swelling in horizontal in between both the groups

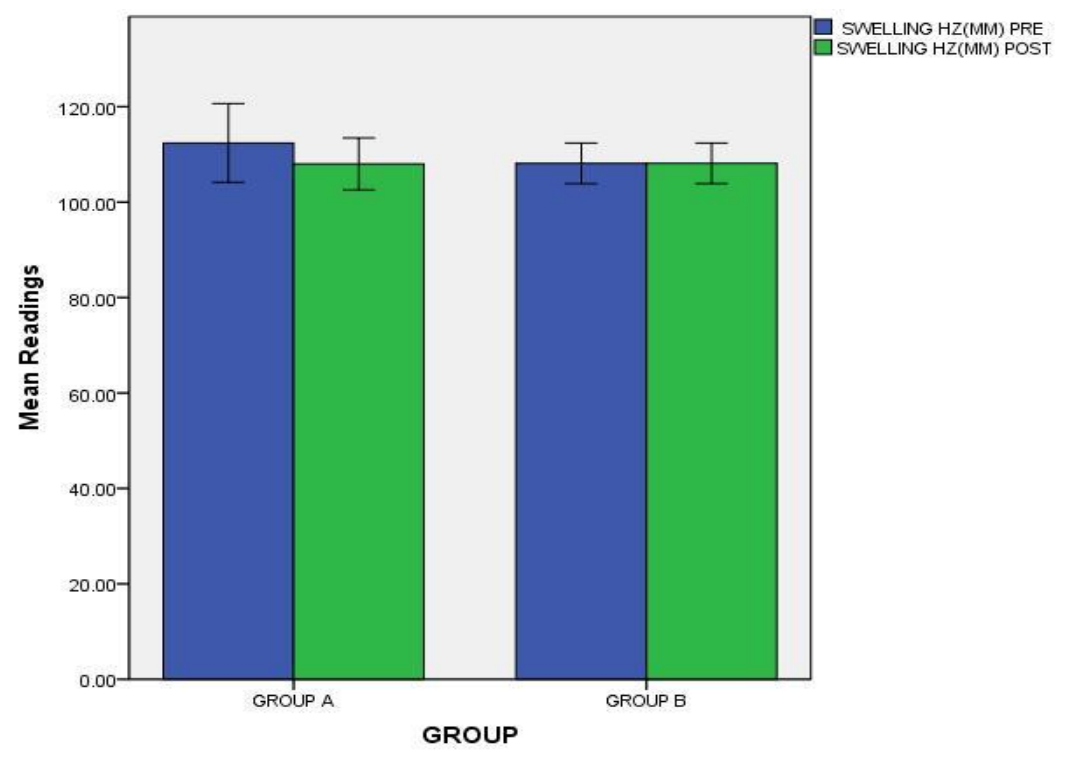

Table 7

Comparison of swelling in vertical in between both the groups

\begin{tabular}{|c|c|c|c|c|c|c|c|c|}
\hline$N=15$ & GROUP & Mean+Std & $\begin{array}{l}\text { Std. } \\
\text { Error } \\
\text { Mean }\end{array}$ & $\begin{array}{l}t \\
\text { test }\end{array}$ & $\begin{array}{l}D \\
f\end{array}$ & $\begin{array}{l}P \\
\text { Value }\end{array}$ & $\begin{array}{l}\text { Mean } \\
\text { Differ } \\
\text { ence }\end{array}$ & $\begin{array}{l}\text { Std. } \\
\text { Error } \\
\text { Differer } \\
\text { ce }\end{array}$ \\
\hline \multirow{2}{*}{$\begin{array}{l}\text { SWELLIN } \\
G V C(M M) \\
P R E\end{array}$} & GROUPA & $111.6+10.12$ & 2.613 & \multirow{2}{*}{$\begin{array}{l}1.1 \\
74\end{array}$} & \multirow{2}{*}{$\begin{array}{l}2 \\
8\end{array}$} & \multirow[b]{2}{*}{$0.25 \mathrm{NS}$} & \multirow[b]{2}{*}{3.733} & \multirow[b]{2}{*}{3.181} \\
\hline & GROUP B & $107.87+7.03$ & 1.815 & & & & & \\
\hline \multirow{2}{*}{$\begin{array}{l}\text { SWELLIN } \\
G V C(M M) \\
P O S T\end{array}$} & GROUPA & $107.34+8.304$ & 2.144 & \multirow[b]{2}{*}{$\begin{array}{l}0.1 \\
90\end{array}$} & \multirow{2}{*}{$\begin{array}{l}2 \\
8\end{array}$} & \multirow{2}{*}{$\begin{array}{l}0.851 N \\
S\end{array}$} & \multirow{2}{*}{0.533} & \multirow[b]{2}{*}{2.809} \\
\hline & GROUP B & $107.87+7.03$ & 1.815 & & & & & \\
\hline
\end{tabular}

Figure 7.1

Comparison of swelling in vertical in between both the groups

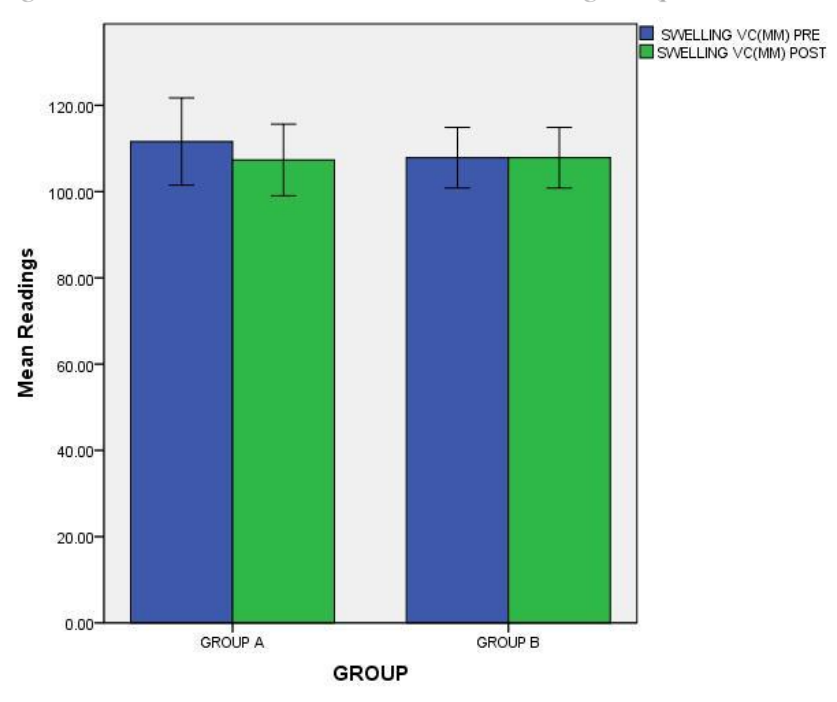


Table 8

Comparison of trismus in between both the groups

\begin{tabular}{|c|c|c|c|c|c|c|c|c|}
\hline$N=15$ & GROUP & Mean+Std & $\begin{array}{l}\text { Std. } \\
\text { Error } \\
\text { Mean }\end{array}$ & $t$ test & $d f$ & $\begin{array}{l}P \\
\text { Valu } \\
e\end{array}$ & $\begin{array}{l}\text { Mean } \\
\text { Diffe } \\
\text { rence }\end{array}$ & $\begin{array}{l}\text { Std. } \\
\text { Error } \\
\text { Differen } \\
\text { ce }\end{array}$ \\
\hline \multirow{2}{*}{$\begin{array}{l}\text { TRISMUS(M } \\
M) P R E\end{array}$} & GROUPA & $34.87+7.06$ & 1.823 & \multirow{2}{*}{-3.491} & \multirow{2}{*}{28} & 0.002 & - & \multirow{2}{*}{1.967} \\
\hline & GROUP $B$ & $41.74+2.866$ & 0.740 & & & $S I G$ & 6.867 & \\
\hline \multirow{2}{*}{$\begin{array}{l}\text { TRISMUS }(M \\
\text { M) POST }\end{array}$} & GROUPA & $41+3.163$ & 0.817 & \multirow{2}{*}{-0.666} & \multirow{2}{*}{28} & 0.511 & - & \multirow{2}{*}{1.102} \\
\hline & GROUP B & $41.74+2.866$ & 0.740 & & & $N S$ & 0.733 & \\
\hline
\end{tabular}

Figure 8.1

Comparison of trismus in between both the groups

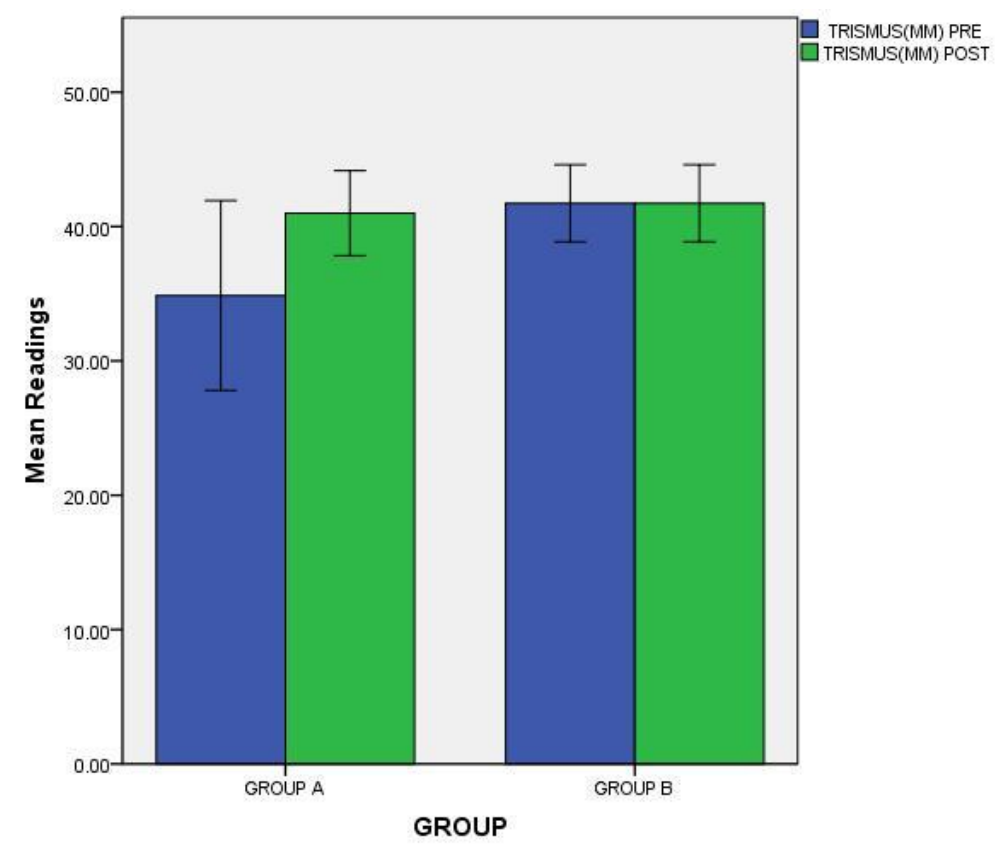

III. Discussion

According to the results of our study, no statistically significant differences were observed in the levels of pain, swelling and trismus between both groups. This clinical finding disagreed with results of Jovanoic et al.,(2004)who expected a thermal insult to tissues stimulated with $100 \mathrm{~mW}$ power density or more but as we shortened the stimulation time, no adverse thermal effects was encountered.

The use of therapeutic laser in the postoperative management of patients having surgical removal of impacted third molars, but without statistically significant differences.(Amarillas et al.,(2010). The low intensity pulsed ultrasound(LIPUS) signal is of low-enough intensity to be considered neither thermal nor destructive.Mechanical signal transduction of LIPUS act as adding mechanical stimuli. The ultrasound serves to take the place of the normal functional loading that would occur under physiological conditions and represent one pathway by which ultrasound may exert its effect on healing. The success of low intensity pulsed ultrasound (LIPUS) and low level LASER therapy(LLLT) to reduce the 
discomforting symptoms after 3rd molar surgeries make it a promising tool for improving orthodontic treatment.

Keywords: Pain,swelling,trismus,ultrasound therapy,laser therapy, third molar surgeries.

\section{REFERENCES}

[1]. Amarillas-Escobar ED, Toranzo-Fernandez JM, Martinez-Rider R, Noyola-Frias MA, Hidalgo-Hurtado JA and Serna, VM. 2010, 'Use of therapeutic laser after surgical removal of impacted lower third molars', Journal of Oral and Maxillofacial Surgery, vol. 68, pp.319-324.

[2]. Berge, TI and Boe, OE. 1994, 'Predictor evaluation of postoperative morbidity after surgical removal of mandibular third molars', Acta Odontol Scand, vol.52, pp.162-169.

[3]. Fisher, SE, Frame, JW, Rout, PG and McEntegart DJ. 1988, 'Factors affecting the onset and severity of pain following the surgicalremoval of unilateral impacted mandibular third molar teeth', Br Dent J, vol.164, pp.351-354.

[4]. Jovanoic, G, Buric, N and Kesic, L. 2004, 'Effect of low power laser

[5]. on postoperative trismus', Facta Universitatis Medicine and Biology, vol.11, no.3, pp.136-138.

[6]. Merry, AF, Gibbs, RD, Edwards, J, Ting, GS, Frampton, C and Davies E. 2010, 'Combined acetaminophen and ibuprofen for pain relief after oral surgery in adults: a randomized controlled trial',Br J Anaesth, vol.104, pp.80-88.

[7]. Miserendino, L, Levy, G and Miserendino, C. 1995, 'Laser interaction with biologic tissues', Lasers in dentistry, pp.39-55.

[8]. Sato, FR, Asprino, L, Araujo, DE and Moraes, M 2009, 'Short-term outcome of postoperative patient recovery perception after surgical removal of third molars', Jounral of Oral and Maxillofacial Surgery, vol.67, pp.1083-1091.

[9]. Tanzer, M, Harvey, E, Kay, A, Morton, P and Bobyn, J. 1996, ' Effect of noninvasive low intensity ultrasound on bone growth into

[10]. porouscoated implants', J Orthop Res, vol.6, no.14, pp.901-906.

[11]. Vegas-Bustamante, E, Micó-Llorens, J, Gargallo-Albiol, J, Satorres-Nieto, M, Berini-Aytés, L and Gay-Escoda, C. 2008, 'Efficacy of methylprednisolone injected into the masseter muscle following the surgical extraction of impacted lower third molars', International Journal of Oral and Maxillofacial Surgery, vol.37, pp.260-263. 